\title{
Regulatory effect of iron regulatory protein-2 on iron metabolism in lung cancer
}

\author{
Z. Cheng, L.L. Dai, Y.N. Song, Y. Kang, J.M. Si, J. Xia and Y.F. Liu \\ Department of Respiratory Medicine, \\ The First Affiliated Hospital of Zhengzhou University, \\ Institute of Clinical Medicine, Zhengzhou, China \\ Corresponding author: Y.F. Liu \\ E-mail: yufengliudoc@163.com
}

Genet. Mol. Res. 13 (3): 5514-5522 (2014)

Received June 13, 2013

Accepted October 25, 2013

Published July 25, 2014

DOI http://dx.doi.org/10.4238/2014.July.25.5

\begin{abstract}
Iron metabolism plays an important role in the pathogenesis of lung cancer. This study aimed to investigate the effect of gene silencing of iron regulatory protein-2 (IRP2) on mRNA and protein expression of transferrin (Tf), transferrin receptor (TfR), and ferritin (Fn) in A549 lung cancer cells. A549 cells were cultured and divided into a liposome control group, a liposome + oligonucleotide (SCODN) control group, and a Lipofectamine + antisense oligonucleotide (ASODN) group. RT-PCR and Western blotting were used to detect mRNA and protein expression of $\mathrm{Tf}$, TfR, and Fn. We found no significant change in Tf mRNA expression among the 3 groups $(\mathrm{P}=0.078)$. TfR and $F n$ mRNA expressions in the ASODN group notably decreased compared to the liposome and SCODN groups $(\mathrm{P}<0.01)$. IRP2 and TfR protein expressions in the ASODN group were significantly lower than in the liposome or SCODN groups $(\mathrm{P}<0.05)$, whereas no significant change in Tf protein expression was observed between the 3 groups $(\mathrm{P}=0.088)$. Fn protein expression in the ASODN group was significantly higher
\end{abstract}


than in the liposome or SCODN group $(\mathrm{P}<0.05)$. IRP2 can regulate the expression of TfR and Fn by changing its own protein expression and thereby regulate iron metabolism.

Key words: Antisense oligonucleotide; Iron regulatory protein-2; Transferrin; Iron protein; Transferrin receptor

\section{INTRODUCTION}

Antisense oligonucleotide (ASODN) microarray technology is one of the most commonly used techniques in basic and applied tumor research. Its basic principle involves generating specific gene-complementing oligonucleotide (ODN) fragments that are designed and synthesized artificially. These fragments combine specifically with the base sequences in a RNA or DNA molecule of a target gene via nucleic acid hybridization, resulting in the specific and effective inhibition of the expression of a gene to achieve the goal of tumor treatment or control (this feature of ASODN can be used for investigating the complementary regulation relationship between genes in basic research). Lung cancer is one of the most common malignant tumors in humans. Its incidence and mortality rates have increased in recent years. Iron, an essential physiological microelement, plays an important role in tumorigenesis (Wang et al., 2011). In squamous cell carcinoma of the head and neck, iron can induce the expression of MMP-9 via the activation of the AP-1-mediated ERK/Akt pathway to drive tumorigenesis (Kaomongkolgit et al., 2008). In addition, iron can exert an influence on the apoptosis of A549 cells in adenocarcinoma of lung (Choi et al., 2009). Therefore, research on the pathogenesis of lung cancer for the therapeutic target by taking iron metabolism as the point of departure will be of great significance. This study aims to explore the role of iron regulatory protein-2 (IRP2), first in the regulation of iron metabolism in lung cancer cells and then in related cellular processes.

\section{MATERIAL AND METHODS}

\section{Design and synthesis of ASODN and RT-PCR primers}

Based on the sequence of IRP 2 mRNA available from GenBank, translation initiation-targeting ASODN was designed. The sequence of $18 \mathrm{bp}$ following IRP2 translation initiation codon (AUG) was 5'-TCCTGCTTTTGGGGCGTC-3'. Meanwhile, a scrambled ODN (SCODN) sequence, 5'-TACGGATCCAATGCGACG-3', was designed as a control in order to analyze the specificity and possible side effects of ASODN. Computer retrieval confirmed that the designed sequences had no homology with any other human gene. Phosphorothioate ODNs were stored with freeze-dried powder for later use.

\section{Cell culture and transfection}

A549 cells in logarithmic phase were digested with $0.25 \%$ trypsinogen. A cell suspension was made, and the concentration was adjusted to $2.5 \times 10^{5} \mathrm{cells} / \mathrm{mL}$. We inoculated $2 \mathrm{~mL}$ cell suspension onto a 6-pore plate, and then cultured at an atmosphere of $5 \% \mathrm{CO}_{2}$ and 
$20 \% \mathrm{O}_{2}$ at $37^{\circ} \mathrm{C}$ for $24 \mathrm{~h}$. A549 cells were divided into liposome (only containing $20 \mu \mathrm{g} / \mathrm{mL}$ liposomes), SCODN, and ASODN groups. The liposome and SCODN groups were used as controls. Liposome-ODN transfection complex was prepared with Lipofectamine 2000 following manufacturer instructions (Invitrogen; Carlsbad, CA, USA) (1640 medium without serum or antibodies was used). The medium was discarded, and then the complex was washed once with 1640 medium without serum or antibodies. Cells were harvested $48 \mathrm{~h}$ after transfection for mRNA extraction.

\section{RT-PCR}

Total mRNA was extracted by Trizol and reversely transcribed into cDNA. PCR amplification was carried out. PCR primers were designed using the Primer 5.0 software based on the gene sequences of transferrin (Tf), transferrin receptor $(T f R)$, ferritin $(F n)$, and $\beta$-actin in GenBank (Table 1). BLAST comparisons showed there were no homologous sequences among them. The PCR volume was $50 \mu \mathrm{L}$. In the amplification conditions, a pre-denaturation was done at $94^{\circ} \mathrm{C}$ for $2 \mathrm{~min}$, followed by 35 cycles at $94^{\circ} \mathrm{C}$ for $30 \mathrm{~s}, 55^{\circ} \mathrm{C}$ for $30 \mathrm{~s}$ and $72^{\circ} \mathrm{C}$ for $2 \mathrm{~min}$, and a final extension at $72^{\circ} \mathrm{C}$ for $6 \mathrm{~min}$. We replaced cDNA with RNase-free water as a negative contrast. We mixed the amplified products with buffer solution and used a $5-\mu \mathrm{L}$ reaction mixture for agarose gel electrophoresis. After ethidium bromide staining, the electrophoresis results were observed using ultraviolet light, recorded using an image recording analytical system, and analyzed by the Quantity One software (Bio-Rad Laboratories, Hercules, CA, USA). The expression of the target gene is reported by the gray value ratio of DNA band of the target gene to that of GAPDH.

\begin{tabular}{|c|c|c|c|}
\hline & Sense strand & Antisense strand & Length (bp) \\
\hline$T f$ & GTCTACATAGCGGGCAAGT & ССTCTTTGTTGTTGGGTTC & 352 \\
\hline$T f R$ & GACTTCACCAGCACCATCAA & CAGCCTTACTATACGCCACA & 262 \\
\hline$F n$ & CGCCAGAACTACCACCAGG & CTTCAAAGCCACATCATCG & 127 \\
\hline$\beta$-actin & CTGGGACGACATGGAGAAAA & AAGGAAGGCTGGAAGAGTGC & 564 \\
\hline
\end{tabular}

\section{Western blotting}

A549 cells in different groups were collected and washed twice with prechilled PBS. The cells were bathed in cell lysate at $4^{\circ} \mathrm{C}$. The cell solution was centrifuged at 15,000 rpm for $1 \mathrm{~h}$, and then supernatant was discarded. Western blot detection was carried out routinely. The samples were transferred to nitrocellulose membranes by electrophoresis at $100 \mathrm{~V}$ for $80 \mathrm{~min}$. The membranes were placed into confining liquid and oscillated slowly at $37^{\circ} \mathrm{C}$ for $2 \mathrm{~h}$. Having been incubated with the primary antibody (Santa Cruz Biotechnology, Santa Cruz, CA, USA) at a dilution of $1: 1000$ at $4^{\circ} \mathrm{C}$ overnight, they were washed with elution buffer solution 3 times $(15,10$, and $5 \mathrm{~min}$, respectively). After elution, the secondary antibody (Invitrogen) at a dilution of 1:4000 was added. ECL coloration was performed. After images were developed, X-ray films were immediately placed into a fixing bath for 5-10 min. The remaining fixer was washed out, and the films were dried at room temperature. Photos were taken for subsequent analysis. 


\section{Statistical analysis}

Data are reported as the means \pm standard error of mean $(x \pm S E)$. Statistical analyses were carried out by the SPSS 13.0 software. The Student $t$-test was used for a pairwise comparison, one-factor analysis of variance was used for comparisons among multiple groups, and the differences were tested by LSD method. $\mathrm{P}<0.05$ was considered to represent a statistically significant difference.

\section{RESULTS}

\section{Morphological changes in A549 cells after transfections}

Significant morphological changes occurred in the ASODN group, including significant heteromorphism, karyopyknosis, nuclear fragmentation, chromatin fragmentation, an increased number of metaphase cells, and the formation of apoptotic bodies, whereas cells in both the liposome and SCODN groups were shuttle-shaped and grew normally (Figure 1A-D).

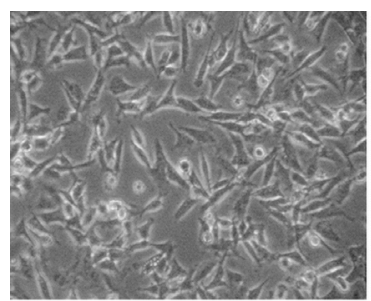

$\mathrm{A}$

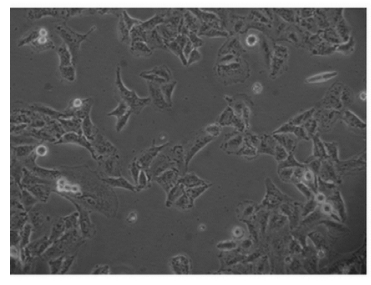

C

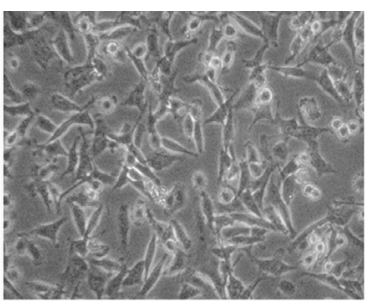

$\mathrm{B}$

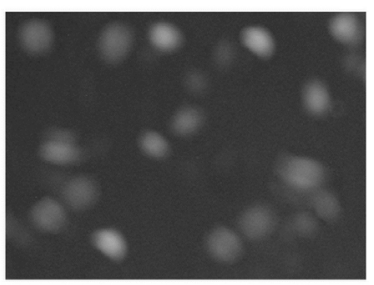

D

Figure 1. Morphological changes of A549 cells after transfection. Cells in liposome and SCODN groups had monomorphism in shuttle-like shape and grew inherently. Cells in the ASODN group had obvious heteromorphism with karyopyknosis, nuclear fragmentation and chromatin fragmentation. A. Cells in the liposome group under inverted microscope (200X). B. Cells in the SCODN group under inverted microscope (200X). C. Cells in the ASODN group under inverted microscope (X200). D. Cells in ASODN under dark field microscope (200X).

\section{$T f, T f R$, and $F n$ mRNA expression}

Changes in mRNA expression in the 3 groups are shown in Table 2 and Figure 2AC. The expression of $T f$ mRNA in the liposome, SCODN, and ASODN groups were $0.440 \pm$ $0.049,0.418 \pm 0.040$, and $0.468 \pm 0.052$, respectively, and no statistical differences were found among them $(\mathrm{F}=2.18, \mathrm{P}=0.078)$ (Figure $2 \mathrm{~A})$. The expression of TfR mRNA in the liposome, SCODN, and ASODN groups were $0.504 \pm 0.052,0.456 \pm 0.096$ and $0.323 \pm 0.032$, respec- 
tively, showing significant differences (Figure 2B) $(\mathrm{F}=20.354, \mathrm{P}<0.001)$. Pairwise comparisons indicated that $T f R$ mRNA expression in the ASODN group was notably lower $(\mathrm{P}<0.01)$, whereas there was no significant difference between the other 2 groups $(\mathrm{P}>0.05)$. Fn mRNA expression in the 3 groups was $0.360 \pm 0.048,0.391 \pm 0.032$ and $0.555 \pm 0.065$, respectively, showing significant differences $(\mathrm{F}=42.785, \mathrm{P}<0.001)$ (Figure $2 \mathrm{C}$ ). Pairwise comparisons indicated that the $F n$ mRNA expression of the ASODN group significantly increased $(\mathrm{P}<$ $0.01)$, whereas there was no significant difference between the other 2 groups $(P>0.05)$. At $48 \mathrm{~h}$ after transfection, there was no significant difference in $T f$ mRNA expression among the 3 groups $(\mathrm{P}>0.05)$. TfR mRNA expression displayed significant differences, among which that of the ASODN group was significantly lower than any other group $(\mathrm{P}<0.01)$. Fn mRNA expression displayed significant differences, among which that of the ASODN group was significantly higher than that of any other group $(\mathrm{P}<0.01)$.

Table 2. Changes of $T f, T f R$ and $F n$ mRNA expressions after transfection $(\mathrm{N}=10)$.

\begin{tabular}{lccc}
\hline Group & Tf & TfR & Fn \\
\hline Liposome & $0.440 \pm 0.049$ & $0.504 \pm 0.052$ & $0.360 \pm 0.048$ \\
SCODN & $0.418 \pm 0.040$ & $0.456 \pm 0.096$ & $0.391 \pm 0.032$ \\
ASODN & $0.468 \pm 0.052$ & $0.323 \pm 0.032^{* \#}$ & $0.555 \pm 0.065^{* \#}$ \\
F & 2.18 & 20.354 & 42.785 \\
P & 0.078 & $<0.001$ & $<0.001$ \\
\hline
\end{tabular}

Pairwise comparisons among three groups: ${ }^{*} \mathrm{P}<0.05$ compared to the liposome group. ${ }^{\#} \mathrm{P}<0.05$ compared to the SCODN group.
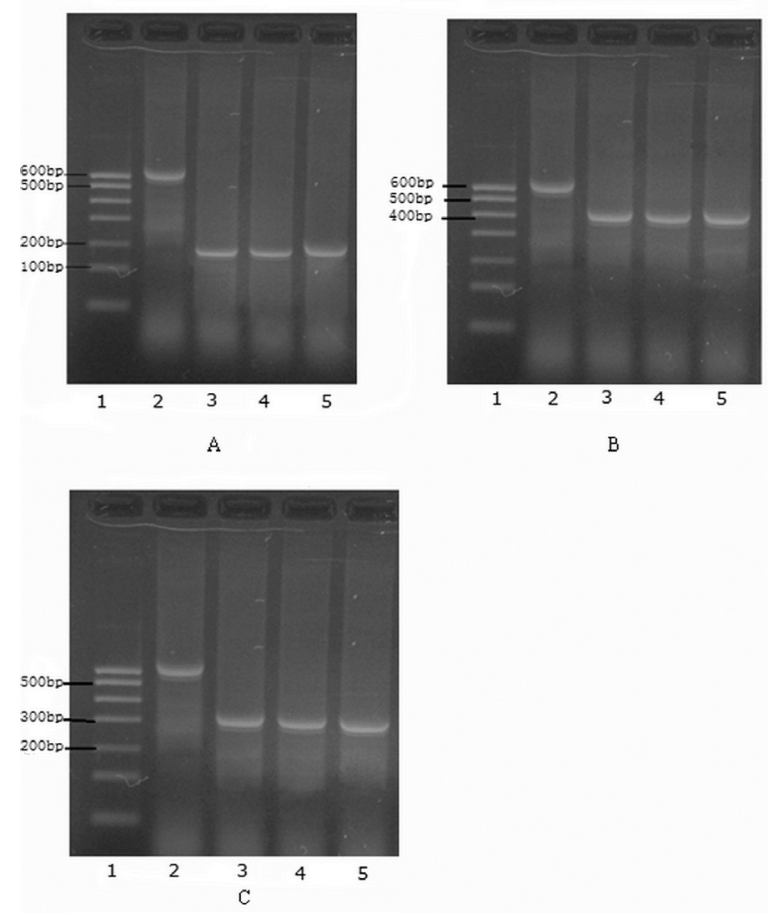

Figure 2. $F n, T f$ and $T f R$ mRNA expressions by RT-PCR after transfection. A. $F n$ mRNA; B. $T f$ mRNA; C. $T f R$ mRNA. Lanes 1, 2, 3, 4, and $5=$ molecular marker, $\beta$-actin, liposomes, SCODN, and ASODN, respectively. 


\section{IRP2, Tf, TfR, and Fn protein expression}

Protein expression patterns in A549 cells are shown in Table 3 and Figure 3A-E. IRP2 protein expression in the liposome, SCODN, and ASODN groups were $0.617 \pm 0.100,0.553$ \pm 0.094 , and $0.120 \pm 0.024$, respectively, displaying significant differences $(\mathrm{F}=113.224, \mathrm{P}<$ 0.001). IRP2 protein expression in the ASODN group was significantly lower than that of the liposome or SCODN group $(\mathrm{P}<0.05)$ (Figure $3 \mathrm{~B})$. Tf protein expression in the 3 groups was $0.651 \pm 0.052,0.630 \pm 0.060$, and $0.593 \pm 0.057$, respectively, showing no significant differences among them $(\mathrm{F}=2.668, \mathrm{P}=0.088)$, but pairwise comparisons showed that there was a significant difference between the ASODN and liposome groups $(\mathrm{P}<0.05)$, whereas no significant difference was found between the ASODN and SCODN group $(\mathrm{P}>0.05)$ (Figure 3C). TfR protein expression in the 3 groups was $0.791 \pm 0.117,0.856 \pm 0.075$, and $0.586 \pm 0.061$, respectively, displaying significant differences $(\mathrm{F}=25.671, \mathrm{P}<0.001)$, and pairwise comparisons between groups showed that there was a significant difference between the ASODN group and the liposome/SCODN group $(\mathrm{P}<0.05)$, whereas no significant difference was found between the liposome and SCODN groups $(\mathrm{P}>0.05)$ (Figure 3D). Fn protein expression in the 3 groups was $0.485 \pm 0.038,0.483 \pm 0.038$, and $0.693 \pm 0.077$, respectively, showing significant differences among them $(\mathrm{F}=49.748, \mathrm{P}<0.001)$, and pairwise comparisons showed that there was a significant difference between the ASODN and liposome/SCODN groups $(\mathrm{P}<$ 0.05 ), whereas no significant difference was found between the liposome and SCODN groups $(\mathrm{P}>0.05)$ (Figure 3E).

Table 3. IRP2, Tf, TfR, and Fn protein expressions after transfection $(\mathrm{N}=10)$.

\begin{tabular}{lcccc}
\hline Group & IRP2 & Tf & TfR & Fn \\
\hline Liposome & $0.617 \pm 0.100$ & $0.651 \pm 0.052$ & $0.791 \pm 0.117$ & $0.485 \pm 0.038$ \\
SCODN & $0.553 \pm 0.094$ & $0.630 \pm 0.060$ & $0.856 \pm 0.075$ & $0.483 \pm 0.038$ \\
ASODN & $0.120 \pm 0.024^{* \#}$ & $0.593 \pm 0.057^{*}$ & $0.586 \pm 0.061^{* *}$ & $0.693 \pm 0.077^{* \#}$ \\
F & 113.224 & 2.668 & 25.671 & 49.748 \\
P & $<0.001$ & 0.088 & $<0.001$ & $<0.001$ \\
\hline
\end{tabular}

Pairwise comparisons between groups: ${ }^{*} \mathrm{P}<0.05$ compared to the liposome group. ${ }^{*} \mathrm{P}<0.05$ compared to the SCODN group.

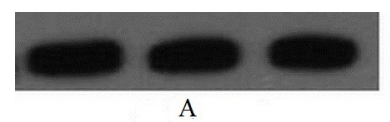

A

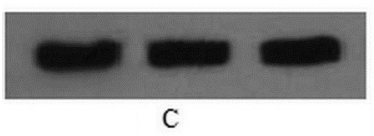

$\mathrm{C}$

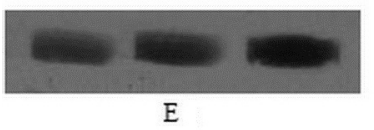

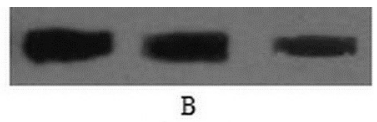

B

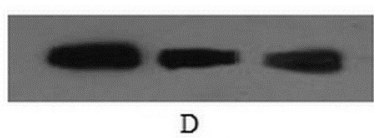

D

Figure 3. IRP2, Tf, TfR, and Fn protein expressions after transfection. A. $\beta$-actin; B. IRP2 protein; C. Tf protein; D. TfR protein; E. Fn protein. Lanes 1, 2, 3, 4, and $5=$ molecular marker, $\beta$-actin, liposomes, SCODN, and ASODN, respectively. 


\section{DISCUSSION}

Lung cancer is a disease associated with high human mortality rates. Although great progress has been made in research and treatment with the development of technology, the overall mortality of lung cancer is still high. Currently, chemotherapy and radiotherapy are the 2 major methods for treating lung cancer. However, due to their poor specificity, many side effects, and unsatisfactory effects efforts to find a more effective drug have become a major focus of lung cancer research and treatment. Therefore, elucidating the molecular basis of a new therapeutic agent and finding its target gene is both a key focus and a major challenge in the study of lung cancer.

Iron is often used as chelating agent and its use for tumor treatment has also been reported (Richardson et al., 2009). Iron use as a chelating agent can upregulate the expression of factors that inhibit tumor growth and metastasis (Le and Richardson, 2004), and the chelation of iron regulates the expression of CyclinD1 via proteasomes, which further regulate the cell cycle and inhibit tumorigenesis (Nurtjahja-Tjendraputra et al., 2007). The ASODN technique is one of the most commonly used established methods in basic and applied tumor research, and it has gradually become the hotspot of research in recent years. This technique first appeared in the 1980s. According to it, a complementary base pair sequence targeting a specific target gene is designed, and then, this sequence selectively and specifically complements with the sequence of an RNA or DNA molecule in the target gene through nucleic acid hybridization to directly interfere or inhibit the production of RNA-encoded proteins, by which to block the expression of the target gene. In essence, the ASODN technique is subjected to a gene therapy by changing an intermediary metabolite of RNA. The ASODN technique can be applied for tumor treatment.

ASODN enters into the cytoplasm of cells via an active transport manner of cell uptake and pinocytosis. However, the transport process is energy-consuming and the transport itself is saturated. Moreover, ASODN can only completely exert its antisense activity when its concentration reaches a certain degree at its target mRNA-combining site in cells. Currently, the most common and widely used method for the introduction of ASODN into cells is the adoption of liposomes to mediate the target transport of ASODN, which have a strong affinity for most membranes with negative charge. In addition, although unmodified ASODN can be absorbed easily by the cell, it can also be degraded easily by nucleases both inside and outside of the cells, and the half-life of unmodified ASODN in blood serum can only last a few hours, or even sometimes $15 \mathrm{~min}$. Compared to the unmodified ASODN, phosphorothioate ASODN has a better solubility, uneasy degradation by nuclease, a longer half-life and a longer lasting effect. Thus, it has more clinically applicable value.

To date, reports on the relationship between iron metabolism and malignant tumors have been released sporadically. A great change in iron metabolism occurs in non-small cell lung cancers (Kukulj et al., 2010). IRP2 can lead to the tumorigenesis through its specific 73-amino acid long regions (Maffettone et al., 2010). The expression spectrum of TfR in normal breast tissue is different from that in breast cancer tissue (Singh et al., 2011). The expressions of TfR 1 and TfR 2 increase in chronic lymphocytic leukemia (Smilevska et al., 2006), and TfR2 is expressed frequently in human malignant tumor cells (Calzolari et al., 2007, 2009; Ikuta et al., 2010). However, neither research on iron metabolism using ASODN technique nor systematic research on iron metabolism-related genes has been reported, to the best of our knowledge. 
IRP2 is an iron-regulating protein and plays an important role in iron metabolism. When there is a change in iron content in extracellular fluid, IRP2, by resorting to the adaptation of its IRE-combining ability (IRE is an iron-response element in Fn and TfR), can regulate the expressions of Fn and TfR to further regulate iron use of the cells, and in so doing, to achieve the iron metabolism-regulating goal (Hausmann et al., 2011). In our study, complexes of modified IRP2-ASODN and liposomes were transfected into A549 cells to observe potential changes in IRP2 expression and, further, to explore the relationships between such changes and the iron metabolism-related genes ( $T f, T f R$, and $F n)$. Our results showed that the expressions of IRP2 mRNA and protein after IRP2-ASODN transfection were notably decreased compared to the liposome and SCODN groups $(\mathrm{P}<0.01)$, indicating that IRP2 ASODN may combine with a specific site to stop the formation of mature mRNA by interfering the capping and splicing of mRNA, or it may combine with mRNA and block access to the correct site of the mRNA molecule for translation, which further affects translation initiation and extension to interfere with the synthesis of the IRP2 protein.

Our results also showed that the expression of TfR mRNA and protein after IRP2 ASODN transfection decreased significantly compared to the liposome or SCODN groups, whereas Fn mRNA and protein levels after transfection increased significantly, indicating that the specific inhibition on IRP2 can influence the expression of TfR and Fn, and such influence may be correlated with the decrease of IRP2 proteins. Meanwhile, our study also found that there was no significant difference in Tf expression before and after IRP2-ASODN transfection, indicating that the inhibition on IRP2 has no notable effect on the expression of Tf, which may be correlated with the mechanism of the inhibition of Tf expression and its role in iron metabolism.

A recent report (Maffettone et al., 2010) showed that wide-type IRP2 can markedly stimulate tumor growth, in which human H1299 lung cancer cells are inoculated into rats and heterograft tumor models are prepared. Our study indicates that IRP2 can regulate the expression of TfR and Fn by changing its own protein expression, thus regulating iron metabolism. Further studies on IRP2 as a new target in lung cancer treatment will be of great importance.

\section{ACKNOWLEDGMENTS}

Research supported by the Health Technology Creative Talents Project of Henan Province (\#2010-52) and and the Natural Science Research Project from the Department of Education of Henan Province (\#2009A320081).

\section{REFERENCES}

Calzolari A, Oliviero I, Deaglio S, Mariani G, et al. (2007). Transferrin receptor 2 is frequently expressed in human cancer cell lines. Blood Cells Mol. Dis. 39: 82-91.

Calzolari A, Finisguerra V, Oliviero I, Deaglio S, et al. (2009). Regulation of transferrin receptor 2 in human cancer cell lines. Blood Cells Mol. Dis. 42: 5-13.

Choi SJ, Oh JM and Choy JH (2009). Toxicological effects of inorganic nanoparticles on human lung cancer A549 cells. J. Inorg. Biochem. 103: 463-471.

Hausmann A, Lee J and Pantopoulos K (2011). Redox control of iron regulatory protein 2 stability. FEBS Lett. 585: 687692.

Ikuta K, Yersin A, Ikai A, Aisen P, et al. (2010). Characterization of the interaction between diferric transferrin and transferrin receptor 2 by functional assays and atomic force microscopy. J. Mol. Biol. 397: 375-384.

Kaomongkolgit R, Cheepsunthorn P, Pavasant P and Sanchavanakit N (2008). Iron increases MMP-9 expression through

Genetics and Molecular Research 13 (3): 5514-5522 (2014)

CFUNPEC-RP www.funpecrp.com.br 
activation of AP-1 via ERK/Akt pathway in human head and neck squamous carcinoma cells. Oral Oncol. 44: 587594.

Kukulj S, Jaganjac M, Boranic M, Krizanac S, et al. (2010). Altered iron metabolism, inflammation, transferrin receptors, and ferritin expression in non-small-cell lung cancer. Med. Oncol. 27: 268-277.

Le NT and Richardson DR (2004). Iron chelators with high antiproliferative activity up-regulate the expression of a growth inhibitory and metastasis suppressor gene: a link between iron metabolism and proliferation. Blood 104: 2967-2975.

Maffettone C, Chen G, Drozdov I, Ouzounis C, et al. (2010). Tumorigenic properties of iron regulatory protein 2 (IRP2) mediated by its specific 73-amino acids insert. PLoS One 5: e10163.

Nurtjahja-Tjendraputra E, Fu D, Phang JM and Richardson DR (2007). Iron chelation regulates cyclin D1 expression via the proteasome: a link to iron deficiency-mediated growth suppression. Blood 109: 4045-4054.

Richardson DR, Kalinowski DS, Lau S, Jansson PJ, et al. (2009). Cancer cell iron metabolism and the development of potent iron chelators as anti-tumour agents. Biochim. Biophys. Acta 1790: 702-717.

Singh M, Mugler K, Hailoo DW, Burke S, et al. (2011). Differential expression of transferrin receptor (tfr) in a spectrum of normal to malignant breast tissues: Implications for in situ and invasive carcinoma. Appl. Immunohistochemistry Mol. Morphol. 19: 417-423.

Smilevska T, Stamatopoulos K, Samara M, Belessi C, et al. (2006). Transferrin receptor-1 and 2 expression in chronic lymphocytic leukemia. Leuk. Res. 30: 183-189.

Wang C, Ding C, Kong M, Dong A, et al. (2011). Tumor-targeting magnetic lipoplex delivery of short hairpin RNA suppresses IGF-1R overexpression of lung adenocarcinoma A549 cells in vitro and in vivo. Biochem. Biophys. Res. Commun. 410: 537-542. 